\title{
A CLINICAL AND RADIOLOGICAL CORRELATION OF THE LESIONS PRODUCED BY CHEMOPALLIDECTOMY AND THALAMECTOMY*
}

\author{
BY \\ GONZALO J. BRAVO and IRVING S. COOPER
}

From the St. Barnabas Hospital for Chronic Diseases, New York

The purpose of this paper is to present the clinical and radiological correlation of 360 intracerebral lesions placed in 300 patients who underwent surgery for the treatment of Parkinson's disease. These cases represent a consecutive series of patients, part of a total of more than 800 basal ganglia operations performed on our service since 1952. Details of the surgical technique employed have already been reported (St. Barnabas Symposium on "Surgical Therapy of Extrapyramidal Disorders", 1956; Cooper, 1956; Cooper and Bravo, 1958a).

The cases selected for this study have been those operated on by using the balloon cannula technique (Bravo and Cooper, 1957) which allows testing of the physiological effects of a temporary lesion before the injection of alcohol and, by creating a small cavity, facilitates the production of a well-circumscribed alcohol lesion (Cooper and Bravo, 1958b). Such lesions, when the alcohol has been mixed with pantopaque, can be visualized radiographically.

In an earlier report we presented the anatomical evidence and the corresponding radiological landmarks obtained as a result of a study carried out in 65 cadaver brains (Bravo and Cooper, 1958). In that report we studied the anatomical location of 120 intracerebral lesions visualized radiographically and confirmed by anatomical studies of each brain. The conclusions of that paper regarding the radiological and anatomical correlations of certain lesions have been confirmed by other investigators (Amador, personal communication). The results of our anatomical study are also in essential agreement with the data that can be obtained from some of the available brain atlases (Stelmasiak, 1956; Spiegel and Wycis, 1952).

The surgical target in the present series of patients was the globus pallidus and/or the thalamus. In our

\footnotetext{
* This work has been supported in part by a grant from the Sister Kenney Foundation, Foundation for Neuromuscular Diseases, and the Office of Vocational Rehabilitation of the Department of Health, Education and Welfare.
}

anatomical studies we were also concerned with the radiological projection of these structures and estimation of limits of safety according to shape and size of the skull, size of the ventricles, and relative position of the foramen of Monro and pineal gland. As a result of continuing investigations a series of intracerebral structures have been radiographically outlined (Figs. 1 and 2) and the landmarks for the two surgical targets, namely the mesial globus pallidus and ventrolateral thalamus, were established (Cooper, Bravo, Riklan, Davidson, and Gorek, 1958). The results of the earlier studies can now be related to radiographically visualized lesions in patients.

In this paper we are going to consider the radiological location of these lesions in terms of the neurological results which they produced. With the information obtained from the cadaver study it is possible to correlate, with a fair degree of accuracy, the anatomical site of these lesions with the observed effects. However, until definite anatomical proof of the structures involved can be shown, when and if the brains of those patients come to post-mortem examination, we should limit ourselves to an evaluation of the clinical and radiological data.

\section{Material and Methods}

Three hundred consecutive patients, all of whom have been operated on by the balloon-cannula alcohol method, are included in this report. The follow-up period ranges from six to 18 months. We have excluded four patients who died in the immediate post-operative period as a result of intracerebral haemorrhage.

Altogether 360 lesions have been plotted in several standard $x$-ray tracings of the skull. For the dimensions of that skull and for the placement of the foramen of Monro and the pineal gland within it we have used a model made with a geometrical mean of the radiographs of all the patients considered. The lesions have been placed in those maps according to their relative position, in terms of the "foramen-pineal line" and in terms of the base line of the skull. The reason why we have chosen 

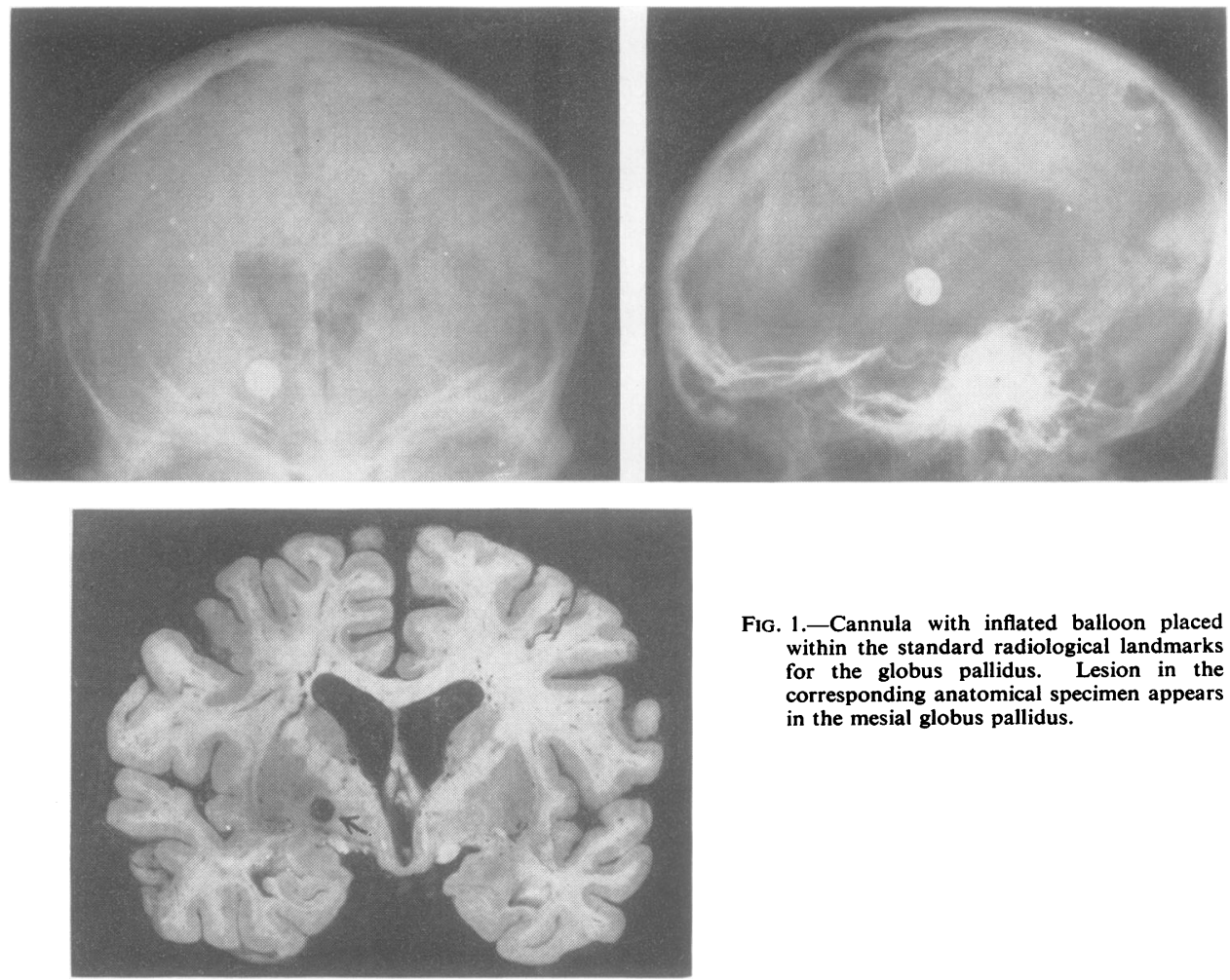

Fig. 1.-Cannula with inflated balloon placed within the standard radiological landmarks for the globus pallidus. Lesion in the corresponding anatomical specimen appears in the mesial globus pallidus.
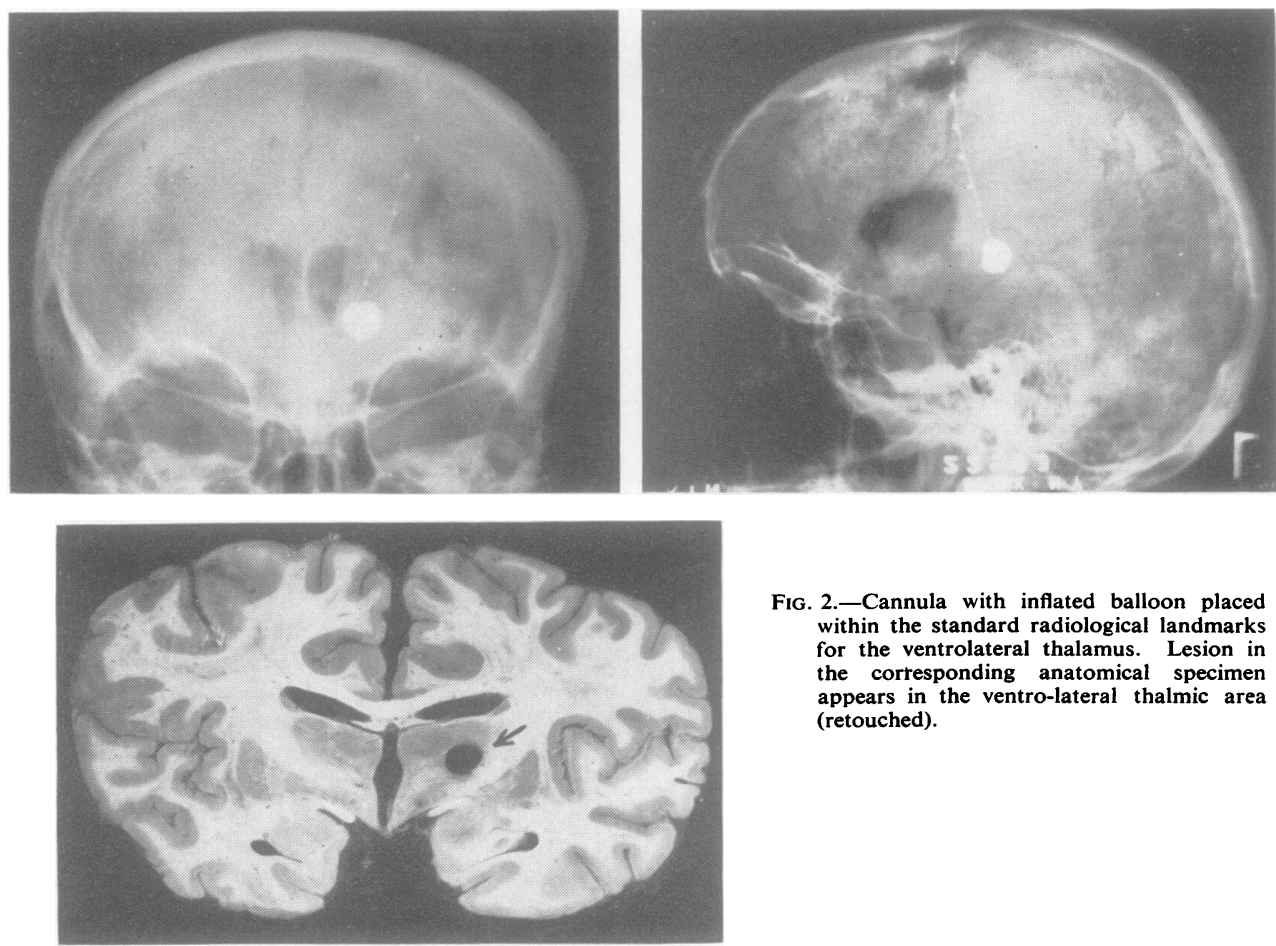

Fig. 2.-Cannula with inflated balloon placed within the standard radiological landmarks for the ventrolateral thalamus. Lesion in the corresponding anatomical specimen appears in the ventro-lateral thalmic area (retouched). 
these reference points, and why we think that relative measurements in relation to them are valid, have been reported elsewhere (Bravo and Cooper, 1958). New data which consider the geometrical positions of the foramen of Monro and the pineal gland as related to the bony structures and relative dimensions of the skull will be the object of a later report.

In order to simplify the presentation, we have chosen as our reference point for the charts the geometrically central area of the lesion, although in some cases the actual lesion extended beyond the boundaries of the circles represented in the accompanying charts. Each point in the charts represents a minimum of three patients with some points corresponding to as many as $\mathbf{4 3}$ patients.

It is evident that, as had been intended at the time of surgery, the lesions fall into two main groups: those directed at the globus pallidus and efferent pallidal pathways, and those aimed at the ventrolateral area of the thalamus. In addition, it became evident that there were several points which showed a fairly constant radiological and clinical correlation outside of the standard limits of the surgical targets. In certain cases, lesions in these adjacent areas produced undesirable results; in other cases, temporary neurological effects with or without accompanying relief of tremor and rigidity were observed.

\section{Results}

The findings of this study have been summarized in five charts. Fig. 5 includes those lesions which affect primarily the globus pallidus and/or its efferent fibres. Fig. 6 includes those lesions which involve different areas of the thalamus and/or efferent or afferent thalamic tracts. We have summarized in Fig. 7 those areas from which serious undesirable effects were obtained. It should be noted that some of those points are based on a very small number of cases and that the complications were, with rare exceptions, temporary. But because of the constant and close relationship between each of those points and the corresponding clinical side-effect, it was considered worthwhile to call attention to it. In Fig. 8 we have isolated the distribution of those lesions causing motor weakness or sensory changes. In some instances the motor weakness was a transitory phenomenon observed in the operating room after inflating the balloon or inserting the cannula. Quick recovery followed the deflation of the balloon or the withdrawal of the cannula and most of these patients were successfully operated on at a later date. In only two instances the lesion thus produced left a residual motor deficit.

Two facts should also be mentioned in relation to the occasional motor deficit associated with chemopallidectomy or chemothalamectomy. (1) The lesion produced by the insertion of the needle or by inflating the balloon, even if incorrectly located, affects only a very small segment of the internal capsule and consequently only transient and temporary

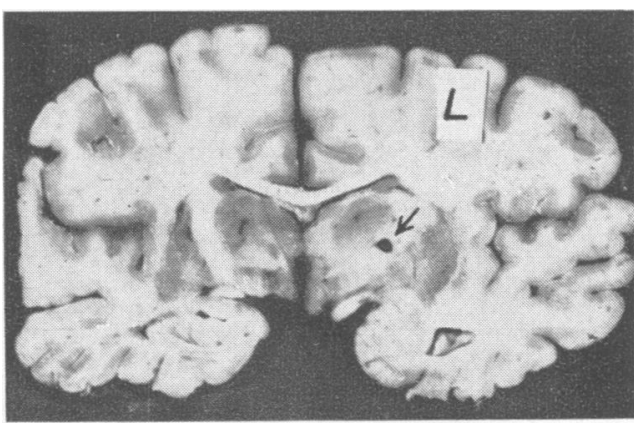

Fig. 3.-Lesion produced in the internal capsule by the inflated balloon.

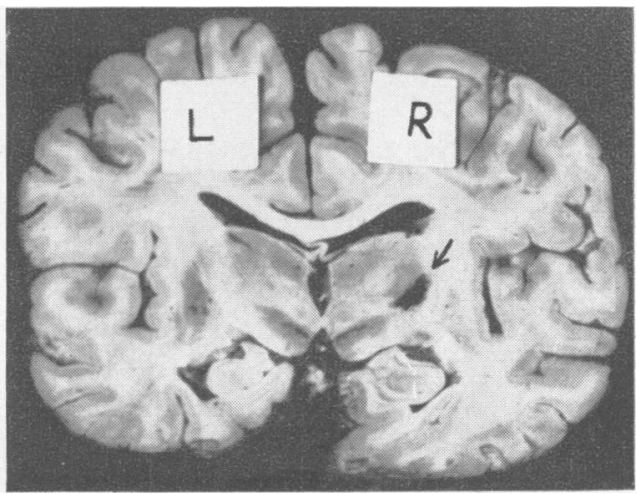

Fig. 4.-Lesion produced by the balloon plus injection of $0.5 \mathrm{ml}$. of alcohol into the ventrolateral thalamus. The lesion is sharply outlined against the internal capsule but it does not spread macroscopically among the fibres of the capsule.

effects should be expected to occur (Fig. 3).

When the inflated balloon itself does not produce a motor deficit, it has never resulted in our experience from subsequent injections of alcohol into the same area unless some bleeding occurred. The explanation for this apparently lies in the fact that when the alcohol is deposited in the most lateral area of the thalamus, the thick fibre layers of the capsule at that level constitute a natural barrier and the alcohol necrosis appears to occur medialward into the soft thalamic tissue (Fig. 4).

Fig. 9 corresponds to the lesions in patient No. 297. This single patient summarizes some of the favourable and unfavourable effects produced by three different radiologically visualized lesions.

Pallidal Lesions.-The five different lesions represented in Fig. 5, which includes a total of 67 patients, correspond to the area of the globus pallidus and its efferent fibres. Although some other projection systems will also be involved with most of these lesions, we have to assume, at the present time, that 


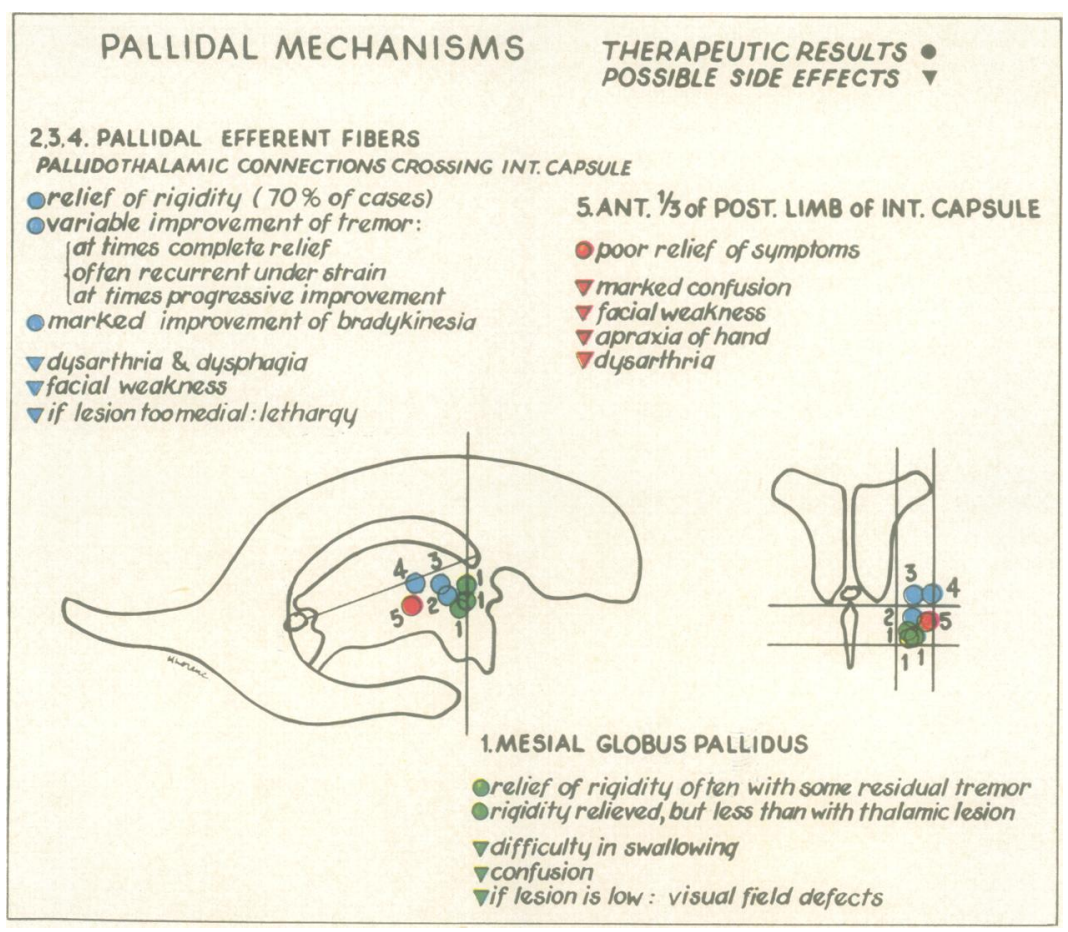

Fig. 5

the effectiveness of these lesions in relieving Parkinsonian symptoms is due to destruction involving the globus pallidus and its efferent fibres, particularly the ansa and fasciculus lenticularis. Lesion No. 1 corresponds to the mesial part of the globus pallidus and it may extend from 10 to $18 \mathrm{~mm}$. from the midline in the antero-posterior radiological projection (Fig. 1). In the lateral view, this lesion may extend from the level of the anterior commissure or anterior border of the foramen of Monro to 4 or $5 \mathrm{~mm}$. behind the posterior margin of the foramen. It should be remembered that as we move posteriorly, part I of the globus pallidus moves away from the midline, so that the mesial globus is not in the same sagittal plane at the level of the anterior commissure, that it is $3 \mathrm{~mm}$. behind the posterior margin of the foramen, but at both levels a pure pallidal lesion can be produced.

Lesion No. 1 has most commonly resulted in alleviation of rigidity and incomplete alleviation of tremor. Occasionally, complete relief of tremor is obtained. No ill effects follow this lesion. Lesion No. 2 affects the innermost part of globus I and the ansa lenticularis. It is possible, also, that some of the anterior fibres of the fasciculus lenticularis might be involved. Lesion No. 2 has generally resulted in a more complete relief of rigidity than lesion No. 1 and the relief of tremor is more often complete or almost complete. Regarding the relief of tremor obtained by this type of lesion, two additional points should be mentioned: (1) In some instances the relieved tremor would transiently reappear under $\mathcal{G}$ strain. (2) In five cases, in which the rigidity was relieved but with marked residual tremor, we observed a gradual, progressive disappearance of the tremor, so that at the end of three months the patients were free of symptoms. Lesion No. 3 involves the antero-ventral segment of the thalamus and the $\stackrel{\mathbb{D}}{\triangle}$ fibres of the fasciculus and ansa as they run into it. This type of lesion has been followed by essentially the same results as lesion No. 2. Lesions Nos. 4 and 5 have in common the involvement of the anterior third of the posterior limb of the internal capsule, through which the fibres of the fasciculus project from the globus to the thalamus. At that level the internal capsule carries fronto-pontine and fronto-bulbar fibres and the anterior segment of the cortico-spinal pyramidal tract. Lesion No. 4, probably because of the involvement of the fasciculus lenticularis, had resulted in improvement of the Parkinsonian symptoms. The results in the cases with the lesion in area No. 5 have been consistently poor, and both lesions Nos. 4 and 5 have been $N$ followed on occasion by transient dysphagia, $N$ dysarthria, and facial weakness. When a lesion of $\mathrm{N}$ this type is placed bilaterally lasting dysphagia and 


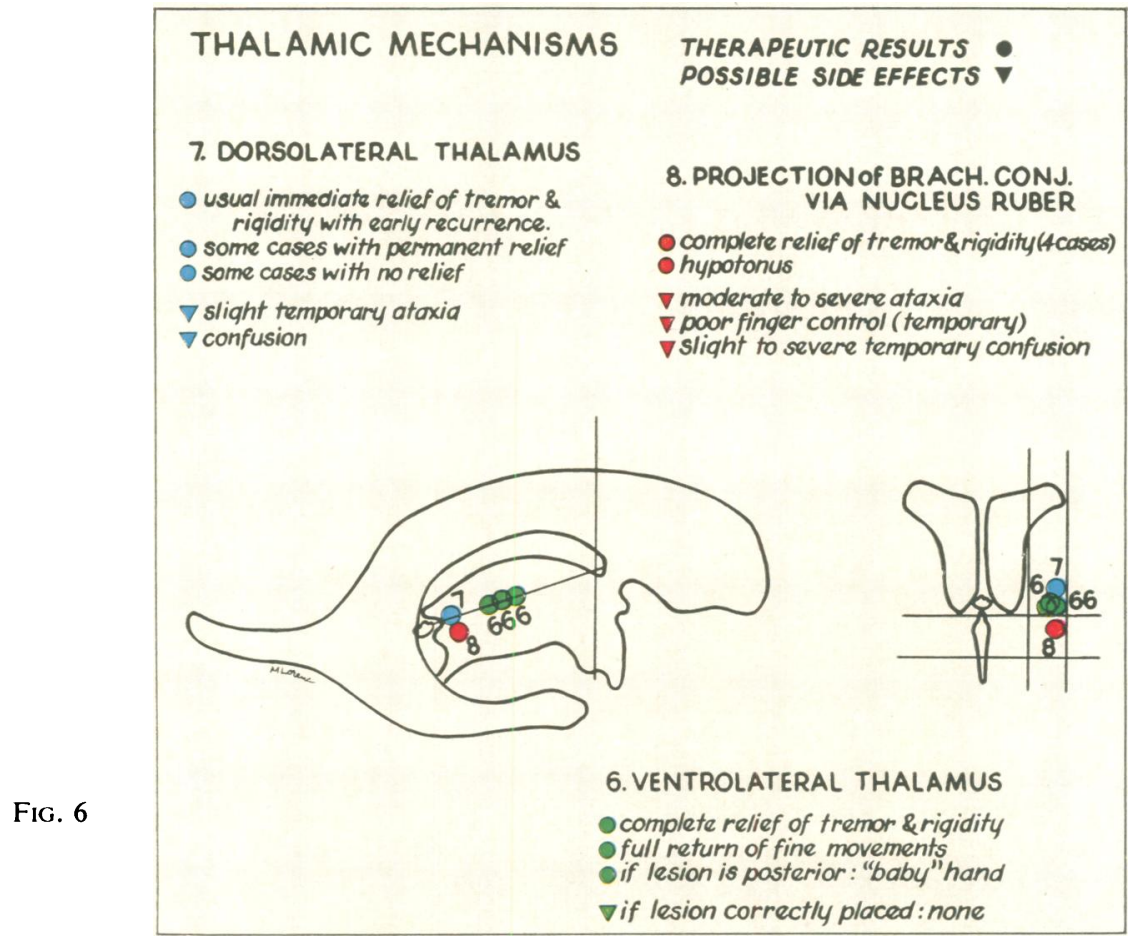

dysarthria may occur. The only other undesirable effects of this group of pallidal lesions have been drowsiness and apathy of a temporary nature.

It should be noted that division within these areas, all of them included within little more than a cubic centimetre of brain, is somewhat artificial. We are speaking in terms of main localization of the lesion and of predominant symptoms. Some small lesions have reproduced rather sharply the effects attributed to a certain localized area; larger destruction, on the other hand, will extend beyond the boundaries of one specific area and the combined effects of lesions in two or three of the described structures would follow.

Thalamic Lesions.-In Fig. 6 we have represented those lesions, 183 in all, which we believe correspond to the ventrolateral region of the thalamus posterior to the anteroventral nucleus. Area No. 6 includes three points, all within the ventrolateral thalamus from which constant results regarding the relief of tremor and rigidity have been obtained. This area seems to start at a point midway between the foramen of Monro and the pineal gland and to extend backwards to a point 7 to $8 \mathrm{~mm}$. in front of the pineal gland. In the antero-posterior projection of the radiograph the centre of the area occupies a point 14 to $20 \mathrm{~mm}$. lateral to the midline (Fig. 2). Details as to the possible structures involved in this type of lesion have been given in a previous anatomical paper. It is not clear at this time if the small variations in the radiological location of that point from which specific response regarding relief of tremor and rigidity can be obtained are due to differences in the radiological projection of the thalamus (due, in turn, to anatomical variations in that particular brain) or if they are related to differences from case to case regarding the physiological mechanisms responsible for the tremor and the rigidity.

Lesions within this area, No. 6, especially in its posterior part, have resulted in relief of tremor and rigidity in practically every case. It should also be noted that we have observed with this type of lesion a return of the fine voluntary movements of the fingers, usually within a period of minutes after placing the lesion. Lesions confined to the limits of area No. 6 have not resulted in any ill effects. In several cases we have observed a certain spatial arrangement within this area, in such a way that following insertion of the needle the relief of the leg symptoms would be obtained from a point postero-caudal in relation to that point affective for the relief of the arm symptoms.

Area No. 7 occupies a zone immediately dorsal and posterior to that of area No. 6. Anatomically, it 


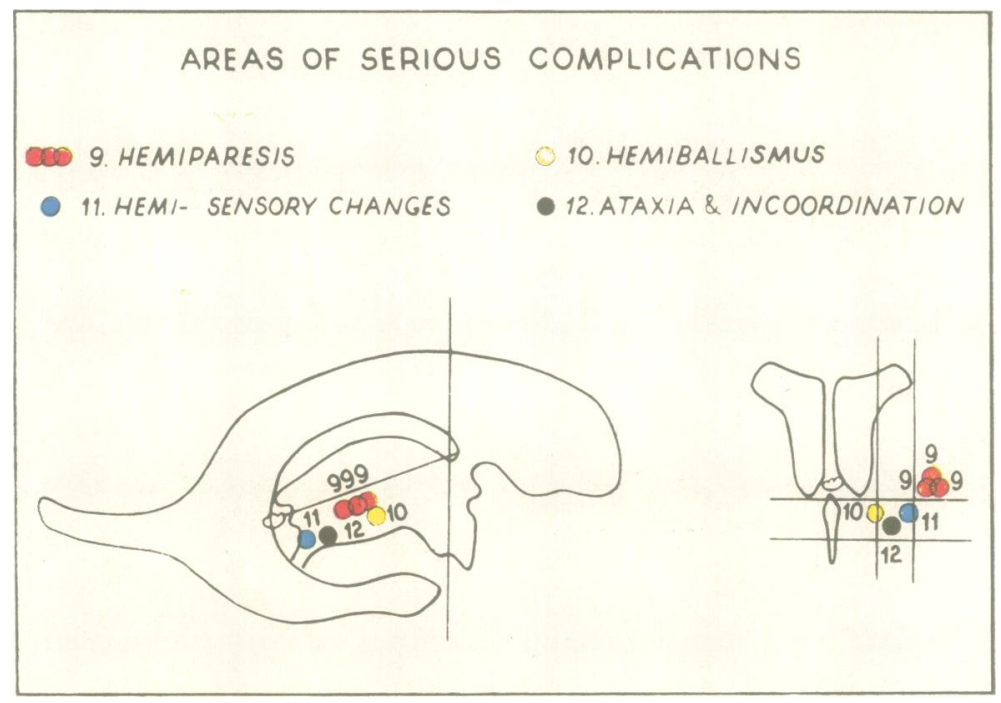

Fig. 7

seems to represent a different zone of the thalamus and, according to our own anatomical studies, areas Nos. 6 and 7 affect different structures and cannot be in the same thalamic nuclei, even if one takes into account the widest possible margin of radiological and anatomical variations. Since the results in some cases with lesions at the level of area No. 7 have been very similar to those obtained at area No. 6, it must be assumed that either two different areas can respond in a similar manner, or that we are interrupting the same pathway at two different levels. This also applies, with certain limitations, to point No. 8, discussed below, and if the concept is widened it would similarly include the favourable pallidal lesions.

Area No. 8 seems to involve the posterior segment of the most ventral part of the lateral thalamus, which receives fibres from Forels fields and, in part, the post-rubral radiation of the brachium conjuntium. Lesions in this area have been followed by relief of tremor and rigidity with a good return of fine voluntary motion, accompanied by a variable degree of temporary incoordination of the limbs, which is manifested by past-pointing, swaying of the raised limb, and a certain clumsiness in manipulation. These signs, minimal in most instances, have cleared within a period of three weeks with maintained relief of tremor and rigidity. Usually, these "cerebellar" signs have been more marked in the medial part of area No. 8. It is possible that the lowermost segment or area No. 8 may also involve projections to or from the substantia nigra.

The thalamic lesions at the indicated points have consistently resulted in an immediate and complete relief of tremor and rigidity. Except for the occurrence of temporary incoordination at point No. 8,0 no ill effect seems to result from these lesions. $A$ 웅 complete looseness or hypotonicity of the affected limbs, without weakness and with full range of $\vec{Q}$ voluntary motion, can be observed within seconds oro $\mathbb{D}$ minutes after inflating the balloon in these sites. It should be noted, however, that the exact point of specific response seems to vary a few millimetres. from patient to patient, and that careful, persistento testing is necessary before considering a placement as final.

Areas of Serious Complications.-Points marked 9 in Fig. 7 are based on the radiographs of seven patients who developed weakness of the contralateral extremities when the needle reached that particular level or when the balloon was inflated in that region. The motor impairment varied from a weakness of grip and heaviness of the arm and leg to a complete flaccid paralysis. All of these patients but one recovered within a period of two to four weeks. In four of the cases, recovery has been complete. Two show residual signs, namely, increased deep tendon reflexes, slight weakness of the hand, and some limitation in the fine movements of the fingers. The remaining patient still has a moderate hemiparesis nine months after surgery. Every one of these patients developed an immediate contralateral sign of Babinski and three of them with lesions in the dominant hemisphere had language difficulties which followed the same favourable evolution as the hemiparesis. It is to be noted that these patients with presumably localized capsular lesions remained 


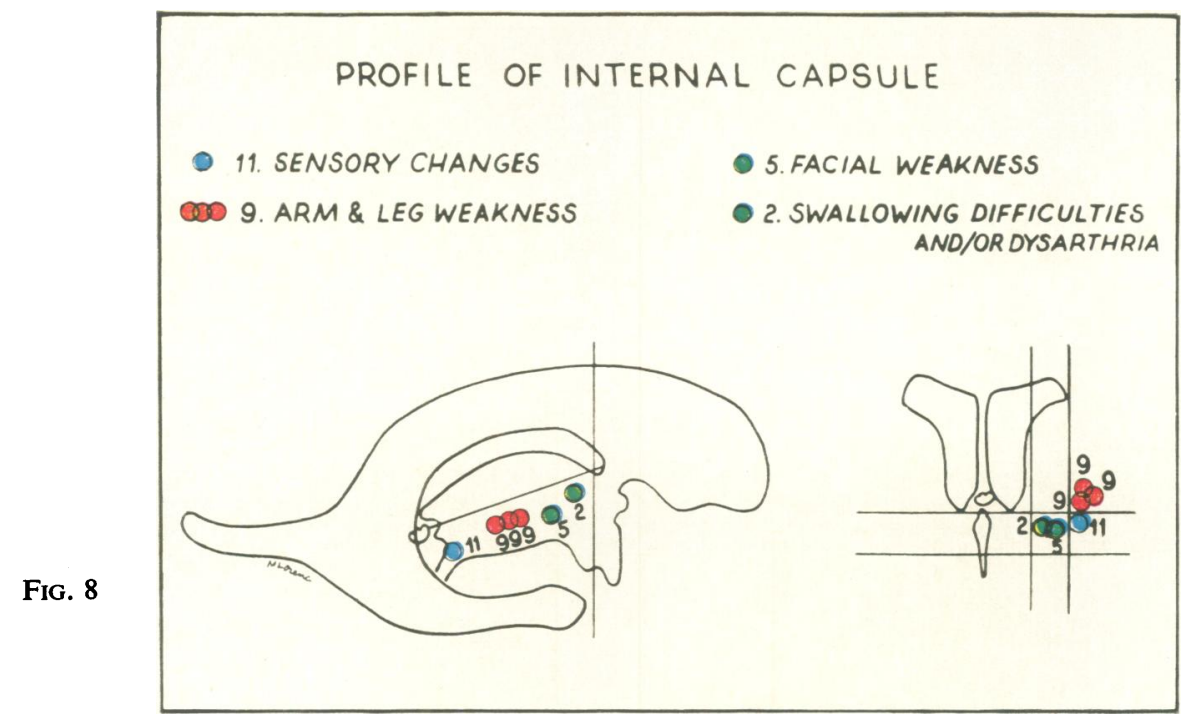

alert and fully conscious in contrast to those patients who developed motor deficits as a result of intracerebral haemorrhage.

This area 9 is immediately lateral to area 6 in our charts. The internal capsule curves at that level around the lateral and caudal aspects of the thalamus. According to our clinical experience, the motor fibres, the lesion of which would cause a hemiparesis, are concentrated in a narrow band behind the mid-point in the foramen-pineal line.

There have been two additional cases in which a hemiparesis was produced on the operating table. These two patients had had previous surgery at the level of point 4 , that is, involving the pallido-fugal fibres and the anterior third of the posterior limb of the internal capsule. No motor deficit resulted at that time, but the symptoms (Parkinsonian tremor and rigidity in one case, and dystonia musculorum deformans in the other) eventually recurred. The patients were operated on again and in both cases the needle entered area No. 9. The hemiparesis which resulted was not of the flaccid type. On the contrary, spasticity developed within a matter of minutes. The hand of the patient could be seen closing and acquiring the posture of a long-standing hemiparetic limb. Both patients improved a great deal with the passing of time, but residual spasticity remained. Whether this phenomenon was due to a double lesion affecting pyramidal and parapyramidal fibres remains open to speculation.

Point No. 11 of this chart represents that area from which sensory abnormalities have been observed. The changes have been in the form of a hypaesthesia and hypalgesia affecting light touch, pin prick, temperature, and pain threshold as measured by galvanic stimulation. These sensory changes have been observed in a total of eight cases. In two of them they were in the form of a hypaesthesia and hypalgesia affecting subjectively the entire contralateral half of the body. Objectively, the sensory changes were more marked on the face and distal part of the extremities. One of these patients has not been available for follow-up examination. The other patient, one year after surgery, still has subjective hemisensory changes, but on objective examinations only patchy areas of hypaesthesia and hypalgesia could be observed on the affected side of the body. The remaining five patients had sensory changes of a similar nature, but limited to the perioral area and to the index finger in one case, and the tips of the second, third, and fourth fingers in the remaining four patients. These changes subsided subjectively and objectively in a period of one to three weeks following surgery.

There have been no cases in which position or vibration senses have been affected.

Lesions at the level of point $\mathbf{1 0}$ have resulted in contralateral involuntary movements in 11 patients. These movements are difficult to describe as a clinical entity and in some cases resemble hemiballistic movements, and in other cases they took the form of a choreoathetosis. The hand alone was involved in five cases, the arm and the leg in three cases, and the entire contralateral side of the body in three cases. Those cases with movements limited to the hand cleared spontaneously in a maximum of two weeks. Those cases with movements in the arm and leg cleared spontaneously between one to three 


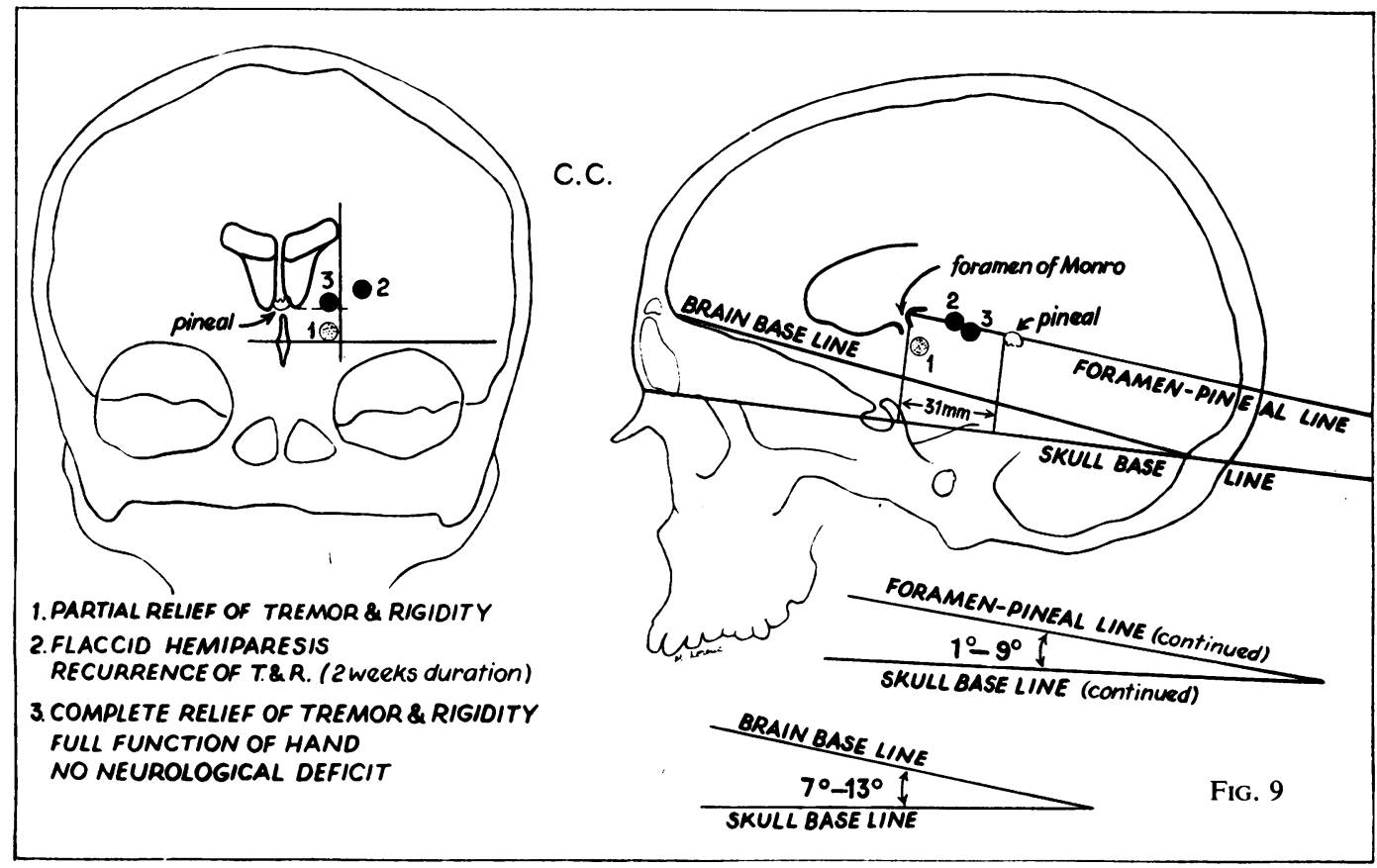

weeks following the onset. Every one of these eight cases eventually had a complete relief of tremor and rigidity in the involved extremities.

Of the three cases with severe choreoathetosis, two of them occurred following bilateral operations, and one of them after a unilateral operation. One of the bilateral cases cleared totally after two weeks of severe choreoathetosis and resulted in complete relief of tremor, rigidity, and bradykinesia. The second of the bilateral cases had mild involuntary movements at the time of discharge from the hospital six weeks after surgery. One patient in this group developed a severe contralateral hemiballismus a few hours after surgery. The involuntary movements slowly subsided and had virtually disappeared within four months at which time the patient died as a result of a carcinoma of the head of the pancreas. Post-mortem examination of the brain revealed a small lesion affecting the fields of Forel and the lateral half of the subthalamic nucleus of Luys. This is probably the same area involved in the other patients with lesions at point 10 . Radiologically, point 10 appears at a level 5 to $9 \mathrm{~mm}$. below the middle third of the foramen-pineal line and 4 to 10 $\mathrm{mm}$. from the midline.

This series of cases of surgically induced involuntary movements will be the object of a detailed report at a later date.

Point No. 12 in Fig 7 represents a region in which surgery has resulted in ataxia, incoordination, an ${ }^{2}$ T marked hypotonus. These effects are not really serious complications. There has been a graduaß recovery in every case and the initially ataxic hypotonic side has returned to normal function witl a maintained relief of the tremor and rigidity.

In Fig. 8 we have summarized some of the points of the previous charts, those from which motor or sensory deficits have been produced, thus giving a radiological and clinical outline of the distribution of the fibres of the internal capsule. Basically this outline is in agreement with the known distribution of anatomical fibres. We have, however, found that those lesions that seem to be responsible for contralateral weakness of the limbs are distributed more posteriorly than one would expect from the standard descriptions in anatomical textbooks. That is, those fibres carrying motor impulses to the contralateral extremities are affected only by lesions at least 15 $\mathrm{mm}$. behind the foramen of Monro at the level of the foramen-pineal line, well behind the so-called genu of the capsule. The question of level of depth of the lesion is important when one considers the distribution of fibres in a sagittal plane because of the double curvature in the internal capsule. This curvature is such that the so-called genu of the capsule becomes displaced posteriorly as the fibres run downward.

As can be seen in Fig. 8, facial weakness has been 
Fig. 10

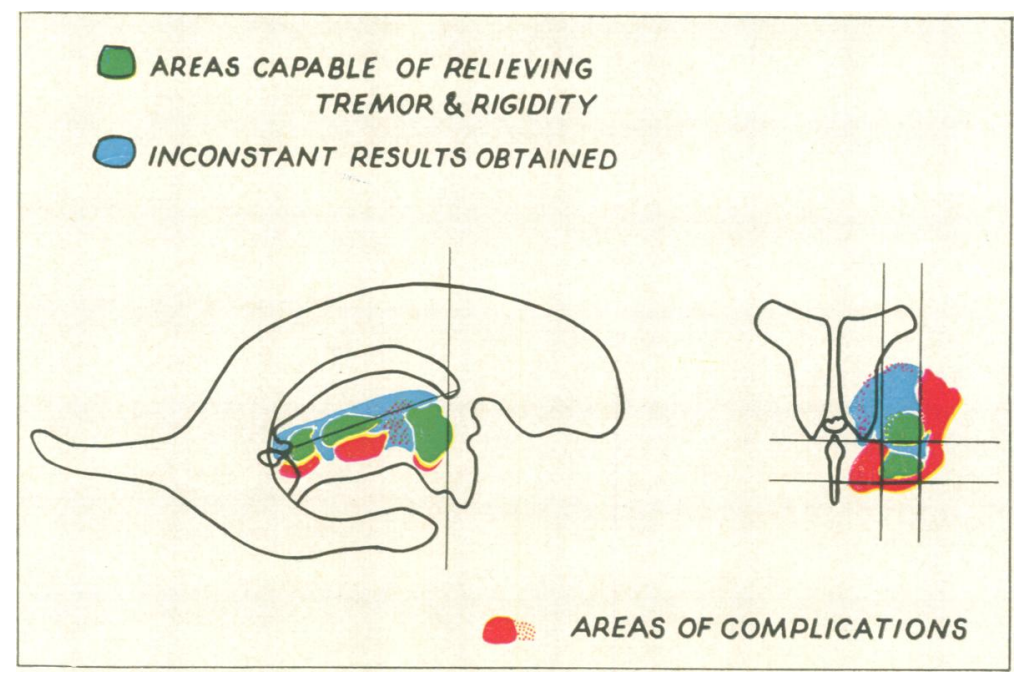

obtained from the anterior segment of the posterior limb of the capsule. When a more posterior segment of the capsule is affected by a lesion the arm will show more marked deficit than the leg without evidence of a specific distribution of fibres. This is the same phenomenon clinically observed after cerebral vascular accidents.

A similar finding of greater susceptibility of the hand and facial fibres have been found at point 11 from which sensory changes were obtained. In most instances the face and the hand, especially the index and middle fingers, showed the more pronounced subjective and objective sensory deficits irrelevant of the special point of lesion.

Fig. 9 emphasizes some of the points of Fig. 6 in relation to the clinical evolution of case No. 297, Mrs. C. C. This 61-year-old woman with Parkinsonism manifested primarily by tremor and rigidity in the right extremities underwent a left transfrontal chemopallidectomy with placing of a well localized lesion at point No. 1 of Fig. 9. Complete relief of rigidity with recurrence of the tremor 10 days after surgery resulted. There were no neurological deficits. Re-operation was decided upon, and the needle was inserted and the balloon inflated at point No. 2 of Fig. 9. Immediately right flaccid paralysis and motor aphasia resulted. The balloon was at once deflated and the needle withdrawn. Progressive recovery of the motor symptoms and aphasis followed and at the end of two weeks the patient was in the same state as before her second operation, tremor included. The third operation was carried out four weeks later. This time the lesion was successfully placed at point No. 3 of Fig. 9 with immediate relief of the tremor and no signs of neurological deficits. This patient has been followed for over one year and has remained free of tremor and rigidity in the right extremities with normal function of the hand and no signs of involvement of the pyramidal tract.*

Fig. 10 is a composite drawing of the favourable, unfavourable, and "neutral" lesions as they appear in the radiographs. Areas from which good clinical results have been obtained without neurological illeffects are represented in green. Lesions which have resulted in neurological complications are represented in red. And finally, those "in-between" areas where results have been inconstant or temporary and the side-effects, for the most part, negligible, are represented in blue.

Two things should be emphasized: first that there is no sharp limit between the green and blue areas, although there seems to be a narrow margin of safety between blue or green and red areas, especially at posterior-external and posterior-inferior levels. Second, that we are dealing with tri-dimensional structures that are represented here in two dimensions, and consequently, there is a superimposition of areas which cannot be shown in the figures. For the representation, preference has been given to the red in both antero-posterior and lateral projections; any lesion which falls within a red area either in the antero-posterior or in the lateral view is likely to be without clinical benefit and result in a neurological complication.

\section{Conclusions}

The most constant and complete relief of both alternating tremor and rigidity is obtained from a

* Since this paper was submitted the same patient has been success fully operated on for her other side. 
lesion in the region of the ventrolateral nucleus of the thalamus. No abnormal sensory, motor, intellectual, or psychological abnormalities result from such a lesion. This lesion is the one of choice for treatment of both tremor and rigidity.

Involvement of cerebellar pathways to the ventrolateral thalamus undoubtedly contributes in some measure to the effectiveness of this lesion in alleviating Parkinsonian tremor and rigidity.

Lesions of the mesial globus pallidus, the efferent pallidal pathways, and the antero-ventral thalamus provide good relief of rigidity but less consistent relief of tremor. However, in some cases, tremor, which was incompletely relieved by a lesion in this complex, will gradually disappear completely within two or three months.

The areas responsible for neurological complications are distributed in a predictable manner, in such a way that those complications can be prevented-or when they occur be explained-in terms of the radiological placing of the lesion.

\section{REFERENCES}

Bravo, G., and Cooper, I. S. (1957). J. Amer. Geriat. Soc., 5, 651.

- (1958). Anatomical and Radiological Correlations in Relation to the Placement of Therapeutic Lesions into the Basal Ganglia. Presented at the New York University Symposium on extra pyramidal disorders on May 15, 1958. To be published.

Cooper, I. S. (1956). The Neurosurgical Alleviation of Parkinsonism. Charles C. Thomas, Springfield, Illinois.

- and Bravo, G. (1958a). J. Neurosurg., 15, 244.

-

-, Riklan, M., Davidson, N. W., and Gorek, E. A. (1958). Geriatrics, 13, 127.

St. Barnabas Symposium on Surgical Therapy of Extrapyramidal Disorders (1956). J. Amer. Geriat. Soc., Vol. IV, Number 12 pp. 1171-1319.

Spiegel, E. A., and Wycis, H. T. (1952). Stereoencephalotomy. Part I: Methods and Stereotaxic Atlas of the Human Brain. Grune and Stratton, New York.

Stelmasiak, M. (1956). Anatomical Atlas of the Human Brain and Spinal Cord. Polish State Medical Publishers, Warsaw. 